\title{
EXCITATION OF A TRANSVERSE QUADRUPOLE-MODE OSCILLATION OF THE ELECTRON BUNCH USING A HIGH-FREQUENCY QUADRUPOLE MAGNET
}

\author{
S. Sakanaka, Y. Kobayashi, T. Mitsuhashi, and T. Obina, Institute of Materials Structure Science, \\ High Energy Accelerator Research Organization (KEK), Tsukuba, Ibaraki 305-0801, Japan
}

\begin{abstract}
In order to investigate the possibility of exciting a transverse quadrupole-mode oscillation of an electron bunch, we carried out an experiment in the Photon Factory storage ring at KEK. While storing single electron bunch, we modulated the betatron tune at a frequency of two-times the fractional betatron frequency, using a high-frequency quadrupole magnet. Then, the transverse quadrupole-mode oscillation could be observed very clearly.
\end{abstract}

\section{INTRODUCTION}

Recently, longitudinal bunch-shape oscillations have been found to be very useful for improving the beam lifetime in the electron storage rings[1-3]. This effect prompted our interest on another bunch-shape oscillation, which is the one in the transverse direction. When a transverse quadrupole-mode oscillation arises, the bunch shape will oscillate either in the horizontal or in the vertical direction at a frequency of two-times the betatron frequency while keeping the center of the bunch still.

Such an oscillation has been studied[4-6] in the synchrotrons for protons or heavy ions. These studies have been found to be useful for investigating such effects as the space charge forces. In the electron storage rings, however, the quadrupole-mode oscillation due to an injection mismatch is damped quickly by the radiation damping. There are usually no powerful mechanism which drives such an oscillation as well. Therefore, the transverse quadrupole-mode oscillation has rarely been studied in the electron storage rings.

In this experiment, we tried to induce the transverse quadrupole-mode oscillation in the Photon Factory (PF) storage ring at KEK.

\section{EXPERIMENTAL METHOD}

\subsection{Excitation of the Oscillation}

We can expect to excite the transverse quadrupolemode oscillation by modulating a betatron tune at a frequency of two-times the fractional betatron frequency. In order to illustrate this mechanism, we consider a betatron motion in a certain (horizontal, in this case) direction. The betatron motion can be approximated by the following equation:

$$
\ddot{x}+\omega_{\beta}^{2} x=0,
$$

where $x$ is the particle displacement, $\omega_{\beta}=\omega_{0} v$ the angular betatron frequency, $\omega_{0}$ the angular revolution frequency, and $v$ the betatron tune. When the betatron tune is modulated at twice the fractional angular betatron frequency (that is, $2 \omega_{0} \Delta v$, where $\Delta v$ is the fractional part of the betatron tune) by a localized focusing force, the motion is essentially described by the following Mathieu equation:

$$
\ddot{x}+\omega_{\beta}^{2}\left[1+\varepsilon \cos \left(2 \omega_{\beta} t\right)\right] x=0,
$$

with

$$
\varepsilon=2 \delta v / v
$$

where $\delta v$ is the peak amplitude of the tune modulation. Here, we have omitted non-resonant terms. Due to a parametric resonance effect, the betatron motion will be excited with a growth rate which is given by

$$
\alpha_{g}=\varepsilon \omega_{\beta} / 4 \text {. }
$$

When the above growth rate exceeds the damping rate, the betatron oscillation grows.

Because the driving frequency is two-times the oscillation frequency, there are two possible oscillations which are out of phase to each other. Each particle among the bunch will take one of the oscillation states randomly. If half of the particles take one of the oscillation state while the other half take the other state, the bunch shape will oscillate at two-times the betatron frequency, resulting in the transverse quadrupole-mode oscillation.

\subsection{High-Frequency Quadrupole Magnet}

An experiment was carried out in the Photon Factory storage ring. The principal parameters of the ring during this experiment were as follows: $E=2.5 \mathrm{GeV}, f_{r f}=$ $500.10926 \mathrm{MHz}, f_{r}=1.60291 \mathrm{MHz}, v_{x}=9.5954, v_{y}=$ $4.2887, \tau_{x}=7.8 \mathrm{~ms}$, and $\varepsilon_{x 0}=3.6 \times 10^{-8} \mathrm{~m} \cdot \mathrm{rad}$, where $E$ is the electron beam energy, $f_{r f}$ the rf frequency, $f_{r}$ the revolution frequency, $v_{x}$ and $v_{y}$ are the horizontal and the vertical betatron tunes, respectively, $\tau_{x}$ the horizontal damping time, and $\varepsilon_{x 0}$ the horizontal natural emittance. Note that the horizontal fractional betatron frequency $\left(f_{\beta x}\right.$ $\left.=f_{r} \Delta v_{x}\right)$ was $954.3 \mathrm{kHz}$.

In order to modulate the betatron tune, we used a highfrequency quadrupole magnet (HFQM)[7], which had been installed in the PF storage ring. This device can 


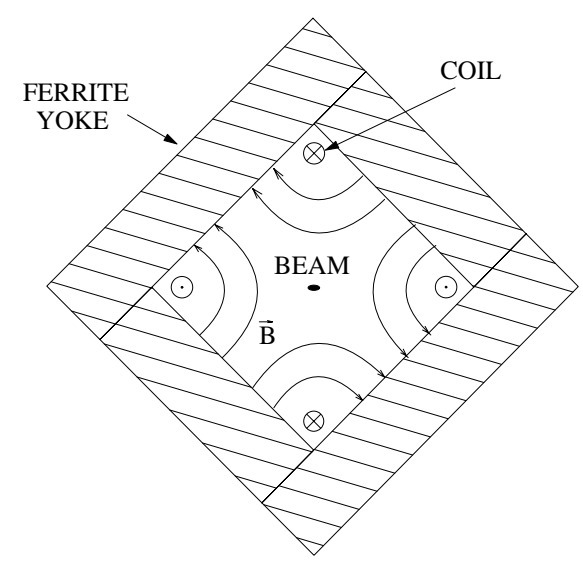

Figure 1: Sketch of the high-frequency quadrupole magnet (HFQM).

Table 1: Principal parameters of the HFQM.

\begin{tabular}{|l|l|}
\hline Parameter & Value \\
\hline Excitation frequency & $1.91 \mathrm{MHz}$ \\
\hline Maximum excitation current & $46 \mathrm{~A}($ peak to peak) \\
\hline Maximum quadrupole strength & $4.1 \times 10^{-4} \mathrm{~m}^{-1}$ (peak) \\
\hline Self inductance & $2.1 \mu \mathrm{H}$ \\
\hline Maximum horizontal tune shift & $3.0 \times 10^{-4}$ \\
\hline
\end{tabular}

produce a weak quadrupole field which oscillates at high frequencies of several MHz. The HFQM is illustrated in Fig. 1. It comprises a single-turn coil and a ferrite yoke, which are placed outside a ceramic duct. The coil is excited resonantly as a part of an LC resonance circuit. We tuned the excitation frequency at twice the fractional betatron frequency $\left(2 f_{\beta x}\right)$ of about $1.91 \mathrm{MHz}$. The principal parameters of the HFQM at this frequency are given in Table 1. With the maximum excitation current of 46 Ap-p, the maximum horizontal tune shift of $3.0 \times 10^{-4}$ (at peak) can be produced. From Eq. (4), this corresponds to a growth rate of about $1500 \mathrm{~s}^{-1}$, which exceeds the radiation damping rate of $128 \mathrm{~s}^{-1}$.

\section{RESULTS}

While storing single electron bunch with low beam currents of about $1.7 \mathrm{~mA}$, we excited the HFQM. All of the octupole magnets were almost turned off. When we increased the excitation current to $8.5 \mathrm{Ap}-\mathrm{p}$, we could observe some beam oscillation with an optical beam profile monitor; this monitor could image the average transverse beam-profiles during many revolutions. In order to observe the beam profile at every turn, we used a dual-sweep streak camera (Hamamatsu C5680). This streak camera could record the longitudinal profile of the bunch, as well as the horizontal one, at the same time. By applying a slow sweep in the horizontal direction of the streak tube, the bunch profiles at successive turns could be recorded.

Figure 2(a) shows the beam image from the streak camera when the HFQM was excited. The excitation current and the frequency were 11.3 Ap-p and 1.9093 $\mathrm{MHz}$, respectively. The beam current was $1.7 \mathrm{~mA}$. In this figure, the beam profiles during 8 revolutions are shown.

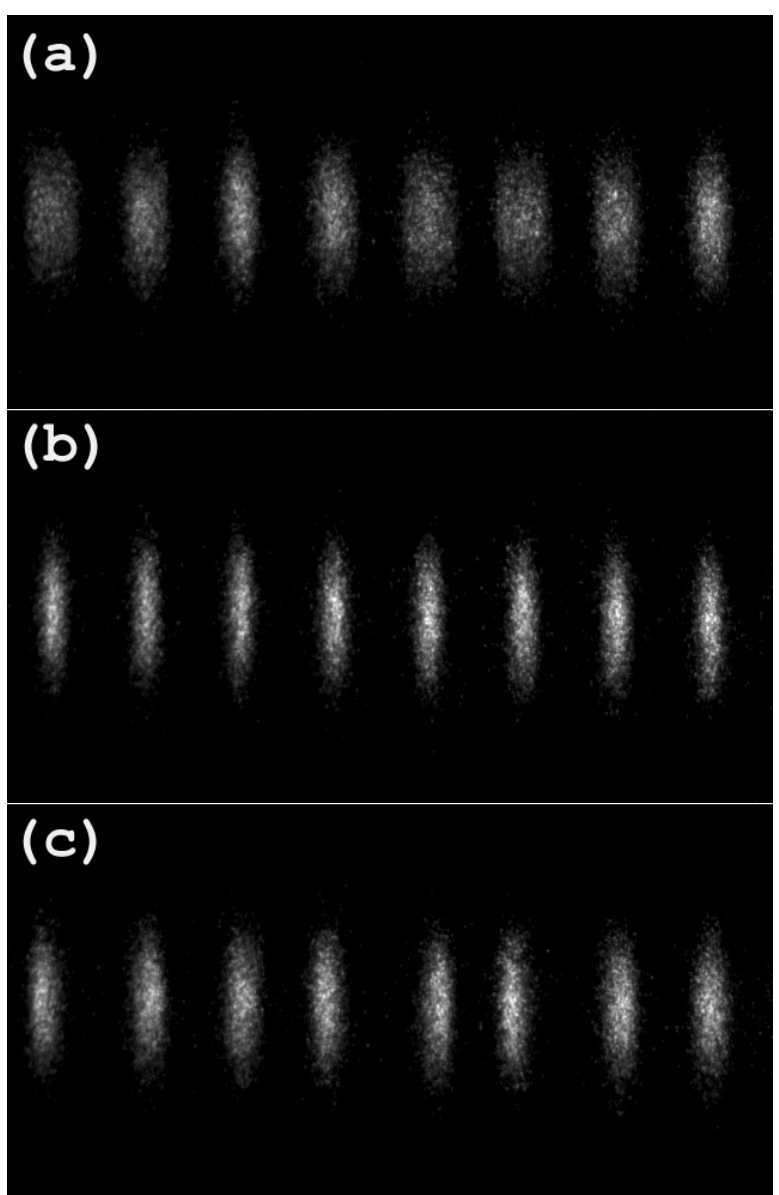

Figure 2: Images from the dual-sweep streak camera, showing both the longitudinal and the horizontal beam profiles during 8 revolutions. Experimental conditions: (a) HFQM on (11.3 Ap-p), (b) without any excitation, and (c) rf knockout on.

The longitudinal beam profiles are shown along the vertical axis (400 ps full scale) while the horizontal profiles (in arbitrary scale) are shown along the horizontal axis. As mentioned above, the slow sweep $(5.13 \mu$ s full scale) in the horizontal direction of the streak camera separated the beam images of multiple revolutions.

It can be seen from Fig. 2(a) that the horizontal beam size oscillated with a period of about 5 revolutions, which corresponded to a frequency of about $300 \mathrm{kHz}$. Because this frequency was close to a frequency of $\left(2 v_{x}-19\right) f_{r}$, the above oscillation is considered to be the horizontal quadrupole-mode oscillation.

For the reference, the beam images, which were measured under different conditions, are shown in Figs. 2(b) and 2(c), respectively. Figure 2(b) shows the beam images when no beam oscillation was excited. The beam profiles during 8 revolutions were very similar to each other. Figure 2(c) shows the beam images when an $\mathrm{rf}$ knockout (oscillating dipole kick) was applied weakly at a frequency of $648.5 \mathrm{kHz}$, that is, a frequency of $\left(1-\Delta v_{x}\right) f_{r}$. In this figure, the bunch profiles are not aligned equidistantly, indicating that the bunch oscillated horizontally as a whole. 




Figure 3: Variations in the horizontal beam profiles during 8 revolutions.
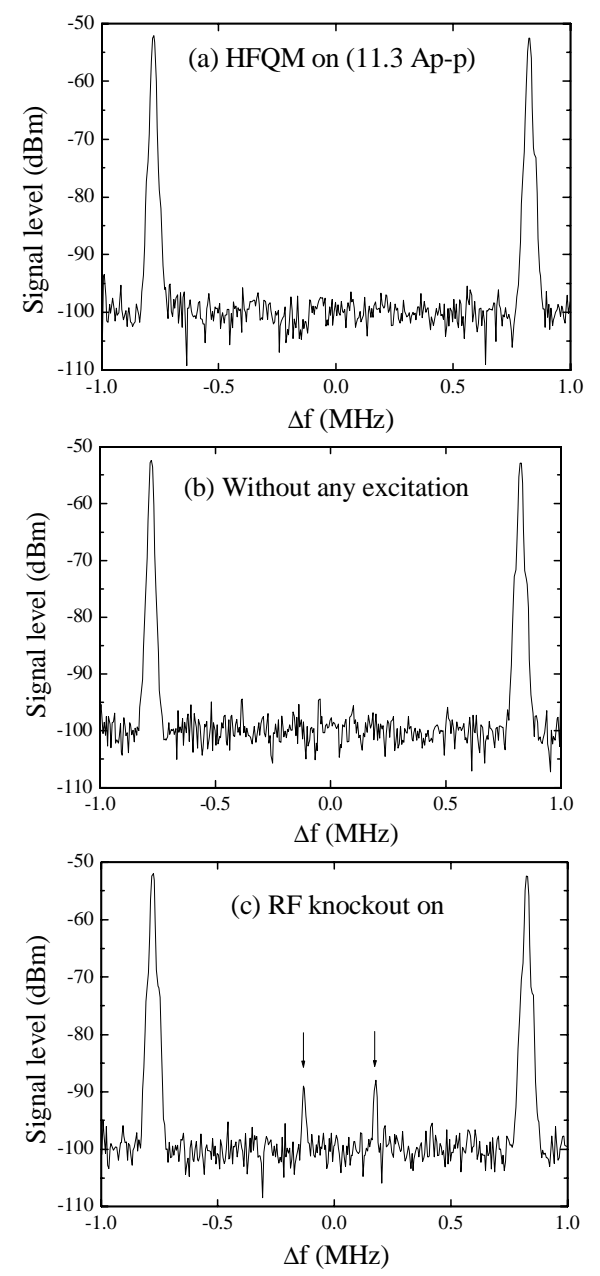

Figure 4: Spectra of the button-type electrode under the conditions of: (a) HFQM on (11.3 Ap-p), (b) without any excitation, and (c) rf knockout on, respectively. Center frequency: $1.001 \mathrm{GHz}$, resolution bandwidth: $10 \mathrm{kHz}$.
Figure 3 shows the variations in the horizontal beam profile, which was obtained by integrating the images in Fig. 2 along the vertical axis. The cases of (a)-(c) in Fig. 3 correspond to Figs. 2(a)-2(c), respectively. In Fig. 3(a), the horizontal beam-size oscillation can be clearly seen.

We also observed the spectra of a button-type electrode signal during this experiment. This electrode was located both lower and side from the beam, and it was sensitive to the horizontal oscillations. Figure 4(a) shows the beam spectrum when the HFQM was excited, that is, under the condition of Fig. 2(a). Apart from two revolution harmonics, no betatron sideband can be seen in this figure, which was very similar spectrum to the one under no excitation (Fig. 4(b)). On the other hand, Fig. 4(c) shows the beam spectrum when the rf knockout was applied. In this case, the betatron sidebands (indicated by arrows) appeared; these sidebands were apart from the revolution harmonics by a fractional betatron frequency $\left(f_{\beta x}\right)$. The above results are reasonable because the button-type electrode would be hardly sensitive to the transverse quadrupole-mode oscillation due to small beam sizes.

\section{CONCLUSIONS}

By applying a quadrupole magnetic field which oscillated at a frequency of two-times the fractional betatron frequency, we could excite a horizontal beam oscillation. This oscillation (Fig. 2(a)) showed the following features: (i) the horizontal beam size oscillated at a frequency of about $300 \mathrm{kHz}$ while keeping the center of the bunch still, and (ii) no betatron sidebands appeared in the button-electrode signal. Because these results are consistent with those expected for the transverse quadrupole-mode oscillation, we can conclude that the observed oscillation should be the horizontal quadrupolemode oscillation. This technique will be useful for studying the wakefield effects on the coherent quadrupole oscillation, or for studying the beam-beam effect in collider rings.

\section{ACKNOWLEDGMENTS}

We would like to thank T. Takahashi for his help in arranging the high-frequency quadrupole magnet. We are also grateful to M. Kobayashi and M. Izawa for their encouragement.

\section{REFERENCES}

[1] Yu. Senichev et al., EPAC'98, p. 1339.

[2] P. Kuske, EPAC'98, p. 1297.

[3] S. Sakanaka et al., Phys. Rev. ST Accel. Beams 3, 050701 (2000).

[4] F. M. Bieniosek and K. Fullett, PAC'95, p. 1942.

[5] M. Chanel, EPAC'96, p. 1015.

[6] T. Uesugi et al., PAC'99, p. 1821.

[7] S. Sakanaka, T. Mitsuhashi, A. Ueda and M. Izawa, Nucl. Instrum. Methods Phys. Res. A 325, 1 (1993). 